Although various risk factors for

\title{
Reducing mortality: an important aim of epilepsy management
}

\section{J W Sander, G S Bell}

\section{Reduction of mortality needs to be paramount in the management of epilepsy}

E pilepsy is often regarded as a benign condition, but there is little doubt that people with epilepsy are two to three times more likely to die prematurely than those without it. ${ }^{1}$ The risk is not uniform over the lifetime of a person with epilepsy, nor across different populations.

Epilepsy is a symptom of a variety of conditions, and the mortality may be different for each condition. In the early years after the diagnosis of epilepsy most deaths are likely to be caused by the background aetiology of the epilepsy-for example, tumours, trauma, degenerative conditions, or cerebrovascular diseases. In this group it is unlikely that treatment of epilepsy will of its own improve the prognosis for life.

People with epilepsy are subject to the same causes of death as the population without epilepsy, but there are some specific ways in which mortality differs. Those with epilepsy may be more likely to die as a result of suicide than the general population, and are more likely to die accidentally. Status epilepticus can occur de novo in people without existing epilepsy, but around half of all cases occur in those already known to have epilepsy. It carries a significant risk of mortality. The population with epilepsy is also at risk from sudden unexpected death in epilepsy (SUDEP). There is controversy about the risk of dying from ischaemic heart disease in people with epilepsy, ${ }^{2-4}$ but mortality is increased for various forms of malignancy. ${ }^{23}$

In an estimation of death in a cohort of people with epilepsy, 30\% of those dying over an eight year period died of accidents, $23 \%$ died suddenly, $16 \%$ died in status epilepticus, and $14 \%$ committed suicide. ${ }^{5}$ Some of these deaths are potentially preventable.

\section{ACCIDENTS}

People with epilepsy are more likely to have accidents and may die as a result. In one study, over one third of subjects with seizures had had at least one injury in the previous 12 months ${ }^{6}$; the type, frequency, and severity of seizures were the best predictors of accidents. Another study in a tertiary referral centre found that over $10 \%$ of subjects had had burns requiring medical attention, and the lifetime number of seizures increased the risk. ${ }^{7}$ Most burns occurred during cooking or showering. It is likely that the same risk factors would apply to fatal accidents as to these non-fatal ones.

One study investigated mortality in a cohort of people who had ever been admitted to hospital with a diagnosis of epilepsy. ${ }^{2}$ Deaths from injury and poisoning were five times more common than expected, and deaths from burning, drowning, and other accidents were also increased. A more recent study looked at the death certificates of people with "epilepsy" anywhere on the certificate. ${ }^{8}$ Injury and poisoning were listed as the underlying cause of death in over $5 \%$ of subjects. The most frequent external causes of death were falling, drowning, and accidental poisoning, usually assumed to be the result of seizures or postictal confusion. Improved seizure control might have reduced these deaths.

Many accidents could be prevented by simple measures, but individual patients must be sufficiently informed to be able to understand the risks and balance them with the desire for a normal lifestyle. This counselling should be part of holistic care, and is best provided by epilepsy specialist nurses.

\section{SUDEP}

SUDEP is defined as "sudden, unexpected, witnessed or unwitnessed, nontraumatic and non-drowning death in patients with epilepsy, with or without evidence for a seizure and excluding documented status epilepticus, in which postmortem examination does not reveal a toxicological or anatomical cause for death." 9 The aetiology of SUDEP is poorly understood. The risk of sudden death for someone with epilepsy in the community is 24 times higher than for someone without epilepsy. ${ }^{10}$
SUDEP have been suggested over the years, few are fully substantiated. Studies in different populations provide different SUDEP rates, and the higher rates reported from studies of people with intractable epilepsy suggest that seizure severity and frequency are important risk factors. ${ }^{11}$ A case-control study found seizure frequency to be the factor most strongly associated with an increased risk. ${ }^{12}$ The use of polytherapy with antiepileptic drugs has also been suggested as a risk factor, but evidence is conflicting. Carbamazepine, phenytoin, and newer antiepileptic drugs have all been suggested as risk factors, but this is not supported by most studies. The evidence suggests that the aim of treatment should be complete seizure suppression, with the smallest effective number and dose of antiepileptic drugs.

\section{STATUS EPILEPTICUS}

Status epilepticus can lead to profound systemic and neurological damage, and is associated with significant short term and long term mortality, ${ }^{13}{ }^{14}$ particularly in adults. The incidence of status epilepticus may be falling. ${ }^{15}$

In children, status epilepticus may be the first epileptic event, but in adults the data are conflicting-between $30 \%$ and $71 \%$ of all adults presenting in status epilepticus do not have pre-existing epilepsy. However, about 5\% of all people with epilepsy have at least one episode of status epilepticus, and a precipitating factor can be found in over half. The most important precipitant is acute antiepileptic drug withdrawal, either because of poor adherence to the drug regimen or under medical supervision. Other precipitants include withdrawal of other drugs or alcohol, infections, other intercurrent illness, or progression of the underlying lesion. It is important to reduce these precipitants where possible. Adherence to the drug regimen should be encouraged, and the risks of not doing so explained. Other precipitants-such as alteration of other drugs and intercurrent illness-may be less easily controlled by the epilepsy specialist, suggesting that the epilepsy service should be readily accessible to other medical professionals for advice.

\section{SUICIDE}

Rates of suicide are increased in people with epilepsy, and suicide in epilepsy may occur at the same rate as that reported among patients with manicdepressive illness. ${ }^{16}$ Various studies give suicide rates of about three times that of

Abbreviations: SUDEP, sudden unexpected death in epilepsy 
the general population, and the rate may be increased even further in people with temporal lobe epilepsy. ${ }^{17}$ Suicide appears to be a serious problem, particularly among those with chronic epilepsy who require treatment in specialty clinics. ${ }^{16}$

One case-control study found a marked increase in relative risk for suicide associated with psychiatric comorbidity and with the use of antipsychotic drugs. ${ }^{18}$ Risk also seemed to increase with high seizure frequency and antiepileptic drug polytherapy, although the associations were not statistically significant. In contrast to this, however, others have found that suicide may occur in patients with long standing complex partial seizures and dysphoric disorder shortly after full control of seizures is achieved. ${ }^{16}$ Epilepsy surgery is often curative and leads to important improvements in quality of life. Risk factors for psychiatric complications after surgery are known, and it is important that patients at risk are identified and managed appropriately. This calls for cooperation between the epilepsy and psychiatric services, particularly for those patients with severe seizures, after achievement of seizure freedom by surgery or new antiepileptic drugs.

\section{ARE THESE DEATHS PREVENTABLE?}

The questions that then arise are: what can we do to prevent these deaths, and what improvements in care are likely to prevent them?

The quest for seizure freedom in people with epilepsy should not be underestimated, as there is increasing evidence that seizure freedom may prevent some deaths. It is also important that people with epilepsy should have access to adequate counselling to reduce the risks of accidents, and to psychiatric services where appropriate.

Anecdote suggests that SUDEP may be more common in the United Kingdom than in other European countries, although comparative data are not available. One difference between the UK and these countries is the number of neurologists serving the population. Recent figures show that the UK has fewer than eight neurologists per million people, while France has 26, the Netherlands 39, and Italy $123 .{ }^{19}$ The distribution of neurologists is not uniform within the UK and there are few with a special interest in epilepsy. Several studies have shown that neurology opinions may contribute useful advice to, or change the diagnosis in, patients previously under the care of non-neurologists, ${ }^{2021}$ and the Association of British Neurologists believes that neurologists who specialise in epilepsy (or other conditions) are better at managing those conditions than neurologists without such specialism. ${ }^{19}$ Access to specialists and specialist clinics was high on the list of suggestions of both patients and neurologists in the Clinical Standards Advisory Group (CSAG) study of services for patients with epilepsy. ${ }^{22}$

People with new-onset seizure should be referred promptly to a consultant with an interest in epilepsy. The NICEcommissioned National Clinical Sentinel Audit into epilepsy related deaths found that only $31 \%$ of patients with newly diagnosed epilepsy saw a specialist within four weeks. ${ }^{23}$ People have died as a result of a second seizure, and, although these deaths may not be readily preventable, investigation and treatment should be taken seriously for those with new onset of seizures. While people with controlled epilepsy may be cared for in primary care, it has been found that primary care services vary from practice to practice, and care is often reactive rather than proactive. ${ }^{24}$ The new GP contract includes additional income for standards reached in epilepsy, and this may help to improve care.

Approximately $30 \%$ of people with epilepsy have seizures that are difficult to control; they should receive optimum treatment to give them the best chance of seizure freedom. However, hospital care is not always optimal. The Sentinel Audit was concerned about many aspects of epilepsy management, which frequently did not meet published criteria. ${ }^{23}$ Optimum treatment for some patients may be surgical, and it has been suggested that all patients with partial epilepsy who fail to respond to two antiepileptic drugs should be referred for consideration of surgery. ${ }^{25}$ The recently publicised case of overdiagnosis of epilepsy in children supports the view that epilepsy services should be organised and based within epilepsy centres and paediatric epilepsy centres, to strengthen primary care, encourage shared care, and allow subspecialisation, as suggested by the CSAG report. ${ }^{22}$ This would also enable the organisation of multidisciplinary care, and encourage cooperation between neurologists, epilepsy specialists, neurosurgeons, psychiatrists, and psychologists. It would support epilepsy specialist nurses, whose roles include information provision, liaison, education, and support, as well as clinical activities. ${ }^{22}$

The Sentinel Audit found that 54\% of adults and $77 \%$ of children had deficiencies in secondary care, and that death was potentially or probably avoidable in $39 \%$ of adults and 59\% of children. ${ }^{23}$ There is, however, no direct evidence that improvements in care would necessarily have prevented any deaths.

Intuitively, it seems likely that optimum use of antiepileptic drugs or neurosurgery to abolish seizure occurrence, or reduce seizures to an absolute minimum, may reduce the excess premature deaths in people with epilepsy. Clearly, both side effects of antiepileptic drugs and patient preference must be considered. However, it also seems likely that most patients, if they fully understand the risks, will choose optimum seizure control.

Being cared for by a neurologist or a paediatrician with an interest in epilepsy, and having access to comprehensive facilities including surgery where appropriate, should improve seizure control and hence reduce the risk of death. We do not have sufficient manpower to achieve this at present, and this warrants urgent rectification.

Evidence based medicine requires that we test all these hypotheses, although if we believe that improving care would result in decreased morbidity and mortality, this may not be an ethical proposition. We may, therefore, never get clear evidence that shortcomings in care may contribute to the premature mortality of people with epilepsy in this country. One option would be to carry out audits of epilepsy related death in countries where care is perceived to be better and where more specialists are in place, to establish whether mortality is decreased in such settings. This may provide some evidence, albeit indirect, on this issue. Nevertheless, the findings of the Sentinel Audit suggest that a significant number of deaths among patients with epilepsy could have been avoided if nationally recommended care standards had been followed. The time has come to make the reduction of mortality paramount in the management of epilepsy.

J Neurol Neurosurg Psychiatry

2004:75:349-351.

doi: 10.1136/jnnp.2003.029223

\section{Authors' affiliations}

J W Sander, G S Bell, Department of Clinical and Experimental Epilepsy, UCL Institute of Neurology, National Hospital for Neurology and Neurosurgery, Queen Square, London $\mathrm{WCl}$, UK

\section{Competing interests: none declared}

Correspondence to: Professor Josemir Sander Box 29, National Hospital for Neurology and Neurosurgery, Queen Square, London WCIN 3BG, UK; Isander@ion.ucl.ac.uk

Received 26 September 2003

Accepted 29 November 2003 


\section{REFERENCES}

1 Bell GS, Sander JW. The epidemiology of epilepsy: the size of the problem. Seizure 2001;10:306-14.

2 Nilsson L, Tomson T, Farahmand BY, et al. Cause specific mortality in epilepsy: a cohort study of more than 9,000 patients once hospitalized for epilepsy. Epilepsia 1997:38:1062-8.

3 Lhatoo SD, Johnson AL, Goodridge DM, et al. Mortality in epilepsy in the first 11 to 14 years after diagnosis: multivariate analysis of a longterm, prospective, population-based cohort. Ann Neurol $2001 ; 49: 336-44$.

4 Shackleton DP, Westendorp RG, Trenite DG, et al. Mortality in patients with epilepsy: 40 years of follow up in a Dutch cohort study. $J$ Neurol Neurosurg Psychiatry 1999;66:636-40.

5 Fukuchi T, Kanemoto K, Kato M, et al. Death in epilepsy with special attention to suicide cases. Epilepsy Res 2002;51:233-6.

6 Buck D, Baker GA, Jacoby A, et al. Patients' experiences of injury as a result of epilepsy. Epilepsia 1997;38:439-44.

7 Spitz MC, Towbin JA, Shantz D, et al. Risk factors for burns as a consequence of seizures in persons with epilepsy. Epilepsia 1994;35:764-7.

8 Jansson B, Ahmed N. Epilepsy and injury mortality in Sweden - the importance of changes in coding practice. Seizure 2002;11:361-70.
9 Nashef L. Sudden unexpected death in epilepsy: terminology and definitions. Epilepsia 1997;38(suppl 11):S6-8.

10 Ficker DM, So EL, Shen WK, et al. Populationbased study of the incidence of sudden unexplained death in epilepsy. Neurology 1998:51:1270-4.

11 Annegers JF, Coan SP. SUDEP: overview of definitions and review of incidence data. Seizure 1999;8:347-52.

12 Nilsson L, Farahmand BY, Persson PG, et al. Risk factors for sudden unexpected death in epilepsy: a case-control study. Lancet 1999:353:888-93.

13 Logroscino G, Hesdorffer DC, Cascino GD, et al. Long-term mortality after a first episode of status epilepticus. Neurology 2002;58:537-41.

14 Simon RP, Pellock JM, Delorenzo RJ. Acute morbidity and mortality of status epilepticus. In: Engel J, Pedley TA, eds. Epilepsy: a comprehensive textbook. Philadelphia: Lipincott-Raven, 1997:741-53

15 Wu YW, Shek DW, Garcia PA, et al. Incidence and mortality of generalized convulsive status epilepticus in California. Neurology 2002:58:1070-6.

16 Blumer D, Montouris G, Davies K, et al. Suicide in epilepsy: psychopathology, pathogenesis, and prevention. Epilepsy Behav 2002;3:232-41.

17 Robertson MM. Suicide, parasuicide, and epilepsy. In: Engel J, Pedley T, eds.

\section{Predicting functional outcome in acute stroke-prognostic models and clinical judgement}

\section{U G R Schulz}

\section{SSV model is a useful research tool}

In their paper (see pp 401), ${ }^{1}$ Counsell et al compare a "six simple variable" (SSV) model, which they developed for predicting outcome after stroke, ${ }^{2}$ with two other simple models and with clinical predictions of stroke outcome, and find that their model is at least as good as the other evaluated predictive systems.

Predictive models of stroke outcome may be useful in epidemiological studies and clinical trials of stroke to stratify cohorts by baseline severity, and they could also guide patient management. A multitude of predictive models already exists, ${ }^{3}$ and one might argue that yet another model is unlikely to add anything new. However, the SSV model is attractive for several reasons: the predictor variables are all based on history and examination and are very easy to collect, and the model has been extensively validated on population and hospital based cohorts, and a clinical trial population. ${ }^{24}$ In this paper, the authors show that the SSV model performs as well or better than two preexisting models. While this is an impor- tant finding, the authors were unable to compare it with any of the other existing models, and we do not know if these might have performed better.

Of more interest is the comparison of the SSV model with clinical judgement. A prognostic model would only be useful in clinical practice if it performed at least as well as a clinician, ${ }^{5}$ and it therefore must be evaluated against clinical judgement. This is the first study to do so, and the authors find little difference between clinical judgement and the statistical model's performance, although they suggest that the model may perform better when compared with the judgement of less experienced clinicians. Even if further studies proved this to be true, the model is still limited to predicting whether a patient will be alive and independent at 1 year. This is only a rather crude outcome measure, because the model cannot take into account type of disability, social circumstances, and other factors, which should play an important part in the physician's prediction of the overall outcome for an individual patient. Even if accurate, the
Epilepsy: a comprehensive textbook Philadelphia: Lippincott-Raven, 1997:2141-51.

18 Nilsson L, Ahlbom A, Farahmand BY, et al. Risk factors for suicide in epilepsy: a case-control study. Epilepsia 2002;43:644-51.

19 Association of British Neurologists. Acute neurological emergencies in adults. London: Association of British Neurologists, 2002.

20 Hillen ME, Sage Jl. Proving the worth of neurologists. Neurology 1996;46:276-7.

21 Steiger MJ, Enevoldson TP, Hammans SR, et al. Influence of obtaining a neurological opinion on the diagnosis and management of hospital inpatients. J Neurol Neurosurg Psychiatry 1996;61:653-4.

22 Clinical Standards Advisory Group. Services for patients with epilepsy. London: Department of Health, 2000

23 Hanna NJ, Black M, Sander JW, et al. The National Sentinel Clinical Audit of epilepsyrelated death: epilepsy - death in the shadows. London: HMSO, 2002

24 Chappell B, Smithson WH. Patient views on primary care services for epilepsy and areas where additional professional knowledge would be welcome. Seizure 1998;7:447-57.

25 Trevathan E, Gilliam F. Lost years: delayed referral for surgically treatable epilepsy. Neurology 2003;61:432-3.

model's clinical usefulness will therefore be limited.

Furthermore, it is important to realise that using prognostic models in clinical practice may already represent a clinical intervention ${ }^{5}$-for example, if only patients with a poor prognosis according to the model were given a specific kind of treatment. Before such models are used in clinical practice, they should therefore not only be shown to be accurate, but also their usefulness should be demonstrated in clinical trials.

The authors quite rightly advise against the use of their model as a guide towards clinical management at present. However, the SSV model is undoubtedly a useful research tool. Epidemiological studies and clinical trials would be much more comparable if they used a single model to stratify according to prognosis or baseline severity rather than each using their individual model. Because of its ease of use, and because of its extensive validation, the SSV model here seems to be a step in the right direction.

J Neurol Neurosurg Psychiatry

2004:75:351-352.

doi: $10.1136 /$ jnnp.2003.032383

Declaration of interest: none

Correspondence to: Dr U Schulz, Stroke Prevention Research Unit, Department of Clinical Neurology, Radcliffe Infirmary, Woodstock Road, Oxford OX2 7NJ, UK; ursula.schulz@clneuro.ox.ac.uk

\section{REFERENCES}

1 Counsell C, Dennis M, McDowall M. Predicting functional outcome in acute stroke: comparison of a simple six variable model with other predictive systems and informal clinical prediction. J Neurol Neurosurg Psychiatry 2004;75:401-5. 
2 Counsell C, Dennis M, McDowall M, et al. Predicting outcome after acute and subacute stroke: Development and validation of new prognostic models. Stroke 2002;33:1041-7.
3 Counsell C, Dennis M. Systematic review of prognostic models in patients with acute stroke. Cerebrovasc Dis 2001;12:159-70.

4 The FOOD Trial Collaboration. Performance of a statistical model to predict stroke outcome in the context of a large, simple, randomized controlled trial of feeding. Stroke 2003;34:127-33.

5 Wyatt JC, Altman DG. Prognostic models: clinically useful or quickly forgotten? BMJ 1995;311:1539-41.
Early changes in parkinsonian brain

\section{Early pathological changes in the parkinsonian brain}

\section{K Abe}

\section{DT-MRI may be able to detect early pathological changes in cerebral white matter}

$\mathrm{T}$ he short report published in this issue by Yoshikawa et al (pp 481) sheds new light on the clinical use of diffusion tensor MRI (DT-MRI) in detecting early pathological changes in the parkinsonian brain. ${ }^{1}$

With the advent of neuroimaging, various techniques have allowed clinicians to monitor the progression of neurological disease, including the parkinsonian diseases. In Parkinson's disease, a representative parkinsonian disease, there is impairment in the nigrostriatal system. Conventional neuroimaging techniques, such as magnetic resonance imaging and computed tomography, can only demonstrate atrophy and intensity changes in the brain, and these are not detectable until after clinical features have become evident. Functional neuroimaging techniques, including single photon emission computed tomography (SPECT) and positron emission tomography (PET), can detect dopaminergic neurone or receptor changes. Morrish et al, ${ }^{2}$ using ${ }^{18} \mathrm{~F}$-dopa PET, showed that only when ${ }^{18} \mathrm{~F}$-dopa uptake in the dorsal caudal putamen is reduced to $50 \%$ of normal do affected individuals became symptomatic. Thus PET may detect early pathological changes. However, the high cost and complications of ionising radiation associated with PET may prevent the routine use of this technique for detecting the early pathological changes in parkinsonian brains. On the other hand, it is well known that the nigrostriatal projection is impaired early in the course of the disease. Thus detecting these impairments may be critical in allowing an early diagnosis. However, most neuroimaging techniques have not been able to detect subtle changes in the cerebral white matter.

DT-MRI has the potential to detect early pathological changes in the cerebral white matter. ${ }^{3}$ Diffusion in the cerebral white matter has anisotropy that becomes evident with disease progression. DT-MRI can measure anisotropy per pixel and can provide information about fibre tracking in vivo. Yoshikawa et al measured fractional anisotropy (FA) in the nigrostriatal projection in Parkinson's disease patients, and compared measured FAs with those of normal controls and of patients with progressive supranuclear palsy, assuming the loss of FA might parallel the neuronal changes. This assumption may be open to discussion, but DT-MRI in combination with other magnetic resonance images may provide clinicians with information about ongoing pathological changes in neurodegenerative diseases.

In further applications of DT-MRI, its potential limitations need to be recognised. These come either from the tracking algorithm or from the DT acquisition. For example, the relatively low resolution of DT-MRI scans may lead to substantial partial volume effects and averaging of fibres with different orientations within a pixel. The singularity problem can be remedied by using smaller voxels for tracking small and interwoven fibre bundles. However, if the voxel contains two or more distinct populations - as may be the case when studying basal structures-reducing the voxel size does not help. ${ }^{4}$ With the concerns raised above about DT-MRI, reports of fibre tracking should be interpreted carefully. Nevertheless, DTMRI has the potential to provide unique quantitative and qualitative information in visualising and studying fibre tract architecture.

J Neurol Neurosurg Psychiatry 2004;75:352. doi: 10.1136/jnnp.2003.031880

Correspondence to: K Abe, Department of Neurology, Osaka University Graduate School of Medicine, 2-2 Yamadaoka, Suita, Osaka 565-0871, Japan;

abe@neurol.med.osaka-u.ac.jp

\section{REFERENCES}

1 Yoshikawa K, Nakata Y, Yamada K, et al. Early pathological changes in the parkinsonian brain demonstrated by diffusion tensor MRI. $J$ Neurol Neurosurg Psychiatry 2004:74:481-4.

2 Morrish PK, Sawl GV, Brooks DJ. Regiona changes in [18F]dopa metabolism in the striatum in Parkinson's disease. J Neurol Neurosurg Psychiatry 1998:64:314-19.

3 Conturo TE, Lori NF, Cull TS, et al. Tracking neuronal fiber pathways in the living human brain. Proc Natl Acad Sci USA 1999:96: 10422-7.

4 Bammer $\mathbf{R}$, Acar B, Moseley ME. In vivo MR tractography using diffusion imaging. Eur J Radiol 2003;45:223-4 